\title{
Spindle cell rhabdomyosarcoma of the tongue - A rare entity
}

\author{
M. T. Khadim ${ }^{1}$, A. Asif ${ }^{2}$, Z. Ali ${ }^{3}$, S. Jamal ${ }^{4}$, Rizwan ${ }^{5}$ \\ ${ }^{1}$ Consultant Histopathologist, ${ }^{2}$ Trainee oral Pathology, ${ }^{3}$ Trainee histopathology, ${ }^{4}$ Consultant Histopathologist, ${ }^{5}$ Trainee oral \\ Pathology, Armed Forces Institute of Pathology, Rawalpindi, Pakistan
}

\begin{abstract}
Spindle cell variant of Rhabdomyosarcoma is a rare malignant tumor in adults but even more so in tongue. We report a case of a 17 year old boy who presented with a polypoidal swelling on the tip of the tongue. The mass was excised and the specimen was sent to the Histopathology Department of Armed Forces Institute of Pathology for Histomorphological diagnosis. The histological evaluation showed a malignant spindle cell tumor. On the basis of morphological features and immunohistochemistry findings a final diagnosis of Rhabdomyosarcoma, Spindle cell variant was made.
\end{abstract}

Keywords: Desmin, rhabdomyosarcoma, tongue.

\section{Introduction}

Rhabdomyosarcoma (RMS) comprises a group of soft tissue neoplasms that shares the propensity to undergo myogenesis. 1 There is a bimodal distribution of presentation with an initial peak incidence between 2-5 years of age and a second surge at 10-19 years. ${ }^{2}$ This results in its resemblance with different stages of skeletal muscle development during prenatal life. The annual incidence of RMS is about 8 million children. ${ }^{2}$ It is the most common sarcoma in infants and children and represents $5-15 \%$ of all the solid neoplasms. ${ }^{2}$ Most common head and neck site include the orbit, nasopharynx, paranasal sinuses, cheek, neck, middle ear and larynx. The occurrence of RMS in the tongue is uncommon. ${ }^{2}$ The spindle cell variant of rhabdomyosarcoma (RMS) is commonly encountered tumor in the paratesticular region of children. ${ }^{3}$ Other sites of involvement include the oral cavity, parotid gland, nasopharynx and nasal cavity. Less than $10 \%$ of adult rhabdomyosarcomas are spindle cell variant. 4,5,6 Histologically they show proliferation of spindle shaped cells with pale cytoplasm arranged in interlacing fascicles. ${ }^{7}$ Individual cells have small nuclei and inconspicuous nucleoli. Scattered rhabdomyoblasts are seen. Only few case of spindle cell RMS of tongue have been reported in literature. $2,8,9,10,11$

\section{Case Report}

We report a case of a 17 years old male who presented with a swelling on the tip of his tongue. The swelling had developed over a period of 8 months. The suspected clinical diagnosis was of Myoepithelioma. The lesion was surgically excised and the specimen was sent to the Histopathology Department for histological evaluation. On gross 
M. T. Khadim et al, Spindle cell rhabdomyosarcoma of the tongue - A rare entity

examination the specimen consisted of a yellow white nodular piece of tissue measuring $7 \times 5 \times 3$ $\mathrm{cm}$. On serial slicing the tumor had whorled appearance. Multiple sections of the tumor were taken and than examined. Microscopic picture revealed a tumor composed of fascicles of atypical pleomorphic spindle shaped cells. The cells were arranged in fascicles and focal storiform arrangement. Many typical rhabdomyoblasts having hyperchromatic eccentric nuclei and abundant eosinophilic cytoplasm were seen. The tumor cells were separated by fibrous stroma. Areas of tumor necrosis were also seen. The tumor had infiltrating borders and was involving the circumferential resection margin and invading the muscles. The morphological differential diagnosis included Rhabdomyosarcoma spindle cell variant, Spindle cell variant of Squamous cell carcinoma, Desmoplastic malignant melanoma, Leiomyosarcoma \& Adult Fibrosarcoma. Immunohistochemical markers were applied. Desmin, Myogenin and Myo D1 were positive. Vimentin showed focal positivity. S-100, GFAP and CK AE1/AE3 were found to be negative. The morphological features and immunohistochemistry results favoured the diagnosis of Rhabdomyosarcoma, spindle cell variant.

\section{Discussion}

Spindle cell RMS is a variant of Embryonal RMS composed of elongated spindle cells and associated with a good prognosis. ${ }^{7}$ Spindle cell variant is relatively newer entry, first described in $1992 .{ }^{7}$ It is uncommon and usually found in the paratesticular region in children. ${ }^{3}$ It is an unusual finding in adults and the tongue is a rare site. 4,11 Clinically these tumors may present as bulging, infiltrative, growing soft tissue masses which may be fungating exhibiting pressure effects with difficulty in speech and swallowing. ${ }^{2}$

On gross examination of the resected specimens, the tumor has firm tan yellow color and whorled cut surface resembling leiomyoma. Histologically, the tumor cells present with shapes of different developmental stages of myogenesis. ${ }^{1}$ Key cell to recognize by routine microscopy is the rhabdomyoblast, a cell with an eccentric round nucleus and variable amounts of brightly eosinophilic cytoplasm. Less than $30 \%$ of cases may show skeletal striations. ${ }^{2}$ The mitotic count is usually low. Palmer and Foulkes. 12 described RMS spindle cell variant being composed of elongate spindle cells arranged in tight fascicles with variable amounts of intervening collagen. A group of these lesions were consequently described by Cavazanna et al. ${ }^{7}$ who confirmed their superior prognosis and found that the majority occurred in the paratesticular region. An independent retrospective review by IRS pathologists. 13 also confirmed the observations made by Palmer and noted a tendency for spindle cell rhabdomyosarcomas to occur in paratesticular regions.

Spindle cell rhabdomyosarcomas may arise in adults. ${ }^{4}$ in whom they may have a worse prognosis. According to the series by Nascimento and Fletcher. ${ }^{4}$ they most commonly arose in the head and neck rather than paratesticular soft tissue. In the setting of adult neoplasia, they can pose a 
Journal of College of Medical Sciences-Nepal, 2011, Vol-7, No-4

diagnostic dilemma because of their resemblance to smooth muscle tumors and other spindle cell lesions. ${ }^{14}$ This challenge can be solved by Immunohistochemistry and/or electron microscopic studies. Immunohistochemistry is used extensively to distinguish RMS from other spindle cell lesions. ${ }^{1}$ Myogenin and MyoD1, myogenic transcriptional regulatory proteins expressed early in skeletal muscle differentiation, are considered sensitive and specific markers for RMS and are more specific than desmin and muscle-specific actin and more sensitive than myoglobin.15,16 As the extent of Myogenin expression in RMS is much greater than in non-RMS, it is a very useful marker when interpreted in the context of relevant clinicopathologic data. In our case the clinicopathological picture as well as the Immunohistochemical results corresponds to the diagnosis of spindle cell variant of Rhabdomyosarcoma of the tongue.

\section{Figure 1:}

Inset A: Spindle shaped cell arranged in interlacing fascicles and focal storiform pattern - Haematoxylon and Eosin Inset B: Immunohistochemistry for Myo D1 showing nuclear positivity.

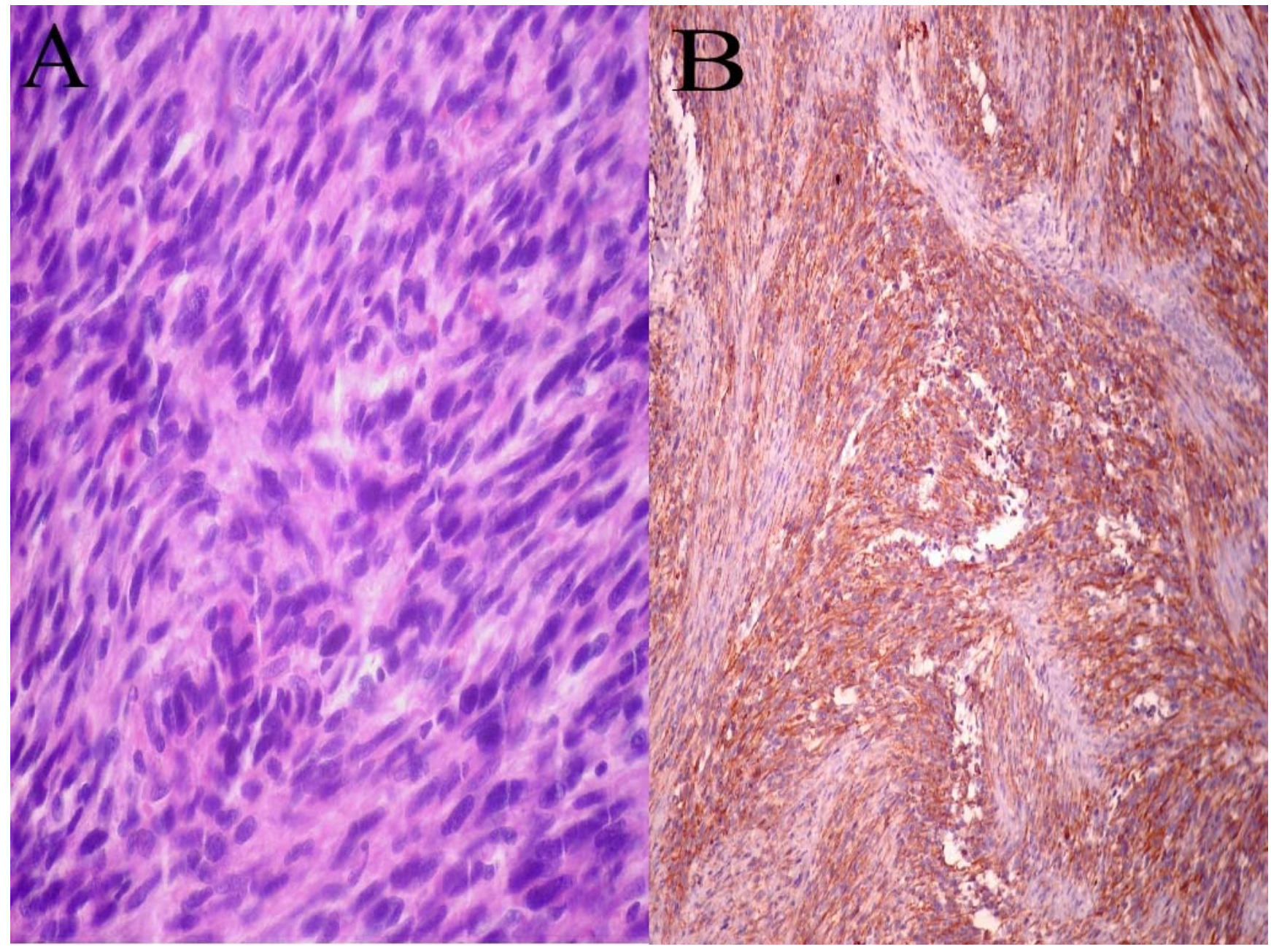


M. T. Khadim et al, Spindle cell rhabdomyosarcoma of the tongue - A rare entity

\section{Conclusion}

Spindle cell variant of Rhabdomyosarcoma is a rare tumor of the tongue. It poses a diagnostic challenge due to its histological similarity with other smooth muscle cell neoplasms. Its frequency in head and neck region is very low. Immunohistochemistry plays key role in its final diagnosis.

\section{References}

1. D.M. Parham, Ellison. Rhabdomyosarcomas. Arch Pathol Lab Med.October 2006; 130:1454-65.

2. G. Gupta, K.S. Bhudwani, R.K. Ghritlaharey,et al. Embryonal Rhabdomyosarcoma tongue in a male child. J Indian Assoc Pediatr Surg. 2006; 11(2):1089.

3. I. Leuschner, W.A. Jr. Newton, D. Schmidt, et al. Spindle cell variants of embryonal rhabdomyosarcoma in the paratesticular region: a report of the Intergroup Rhabdomyosarcoma Study. Am J Surg Pathol. 1993(17):221-30.

4. A.F. Nascimento, C.D. Fletcehr. Spindle cell Rhabdomyosarcoma in adults. Am J Surg Pathol. 2005; 29(8):1106-13.

5. T. Mentzel, C. Kunhen. Spindle cell Rhabdomyosarcoma in adult: Clinicopathological and immunohistochemical analysis of seven new cases. Virchows Arch. 2006; 449(5): 554-60.

6. A. Baldi, G. Nicoletti, G. Colella et al. Embryonal Rhabdomyosarcoma of the tongue in old age. Oral Onco. 2004; 40 (4):450-2.

7. A.O. Cavazzana, D. Schimdt, V. Ninfo et al. Spindle cell Rhabdomyosarcoma: A prognostically favourable variant of Rhabdomyosarcoma. Am J Surg Pathol. 1992; 16(3):229-35.
8. A. Gupta, J. Madallozo, T. Winttin et al. Spindle cell Rhabdomyosarcoma of tongue in an infant: a case report with emphasis on differential diagnosis of childhood spindle cell lesions, Pathol Res Prac. 2004; 200(7-8); 537-43.

9. D.C. Doval, V. Kannan, R.S. Acharaya et al. Rhabdomyosarcoma of tongue. Br J Oral Maxillfac Surg. 1994; 32(3): 183-6.

10. P.S. Libert, S.E. Stool. Rhabdomyosarcoma of tongue in an infant: Results combined radiotherapy and Chemotherapy. Ann Surg. 1973; 178(5): 621-4.

11. R. Kebudi, G.N. Ozmedir. Rhabdomyosarcoma of tongue: A report case and review of literature. Pediatr Hematol Oncol. 2011; 28(1): 60-4.

12. N.F. Palmer, M. Foulkes. Histopathology and prognosis in the second Intergroup Rhabdomyosarcoma Study (IRS II) [abstract]. Proc ASCO. 1983;2:229.

13. W.A. Jr. Newton, E.A. Gehan, B.L. Webber, et al. Classification of rhabdomyosarcoma and related sarcomas: pathologic aspects and proposal for a new classification - an Intergroup Rhabdomyosarcoma Study. Cancer. 1995;76:1073-85.

14. B.P. Rubin, R.P. Hasserjian, S. Singer et al. Spindle Cell Rhabdomyosarcoma (so called) in adults: report of 2 cases with emphasis on differential diagnosis. Am J Surg Pathol 1998; 22(4): 459-64.

15. Cessna, Melissa H. M.D.; Zhou, Holly M.D. et all. Are Myogenin and MyoD1 Expression Specific for Rhabdomyosarcoma?: A Study of 150 Cases, With Emphasis on Spindle Cell Mimics. Am J Surg Pathol. $2001 ; 25(9): 1150-7$.

16. D.M. Parham . Review article. Pathologic Classification of Rhabdomyosarcomas and Correlations with Molecular Studies. Mod Pathol 2001;14(5):506-14. 